\title{
Energy Efficient Model for Data Transmission in WSN
}

\author{
Ashutosh Kumar Choudhary \\ Assistant Professor \\ Amity School of Engineering \&Technology \\ Amity University,Chhattisgarh \\ akchoudhary@rpr.amity.edu
}

\begin{abstract}
Energy efficient routing protocols are regularly cited as effective results for Wireless Sensor Networks (WSNs) routing. In wireless sensor network the patient monitoring is the major issue to solve various health problems. Patient requires prompt $\&$ accurate decisions so that lifesaving therapy can be properly applied. Because of these necessities, ICUs have become broadly recognized in hospitals. These systems work once there is any emergency by using diverse wireless technologies. This system is mainly based on continuous monitoring aspect of patients. This system is designed, developed a reliable, energy efficient patient monitoring system. It is able to send parameters of patient in real time to provide the continuous monitoring and to save the battery life of network. Two important techniques have been used, firstly; the power estimated according to varying distance between sensor nodes. Secondly, sensor nodes are set to sleep state between two consecutive measurements for energy saving in normal operating condition. The timely routine of assigning real time examined parameter to doctor is given maximum importance. The result shows significant improvement in continues patient monitoring aspect as compared to traditional monitoring aspect.
\end{abstract}

\section{INTRODUCTION}

Wireless Sensor Networks (WSN) consists of several sensor enable nodes which are distributed in an environment and use batteries as energy resource. These small sensor nodes, which consist of finding, data processing, and communicating units, result in the idea of sensor networks based on collaborative effort of many nodes. Such sensor nodes could be utilized in home, military, science, and industry applications such as transportation, healthcare disaster recovery, warfare, security, industrial and building automation, and even space exploration. Between a large variety of applications, phenomena observing is one of the key areas in wireless sensor networks and in such systems, you can query the physical quantities of the environment.

As the sensor nodes are tiny and batteries empower gadgets, they have restricted energy which should be used accurately. Thus, the limited sensor resources (in particular, the battery power) are easily over consumed. Thus, the key question in such phenomena observing is conserving the sensor energy, to maximize their life cycle. Most of the approaches tried to response to this challenge and this will be continuing to gain a better solution. Energy constraints are major issue in wireless sensor network. Major energy is used for data collection and transmission.

\section{Problem definition:}

Currently there are many health monitoring systems are available for patients. All these systems are useful only during emergency occurs. Information transmitted to the doctor side only during any abnormality occurs. But the important problem in these systems is 
related to range required for transmitting the information to the doctor side or receiver side. Sometimes it does not provide continues range for transmitting data. Many times, node level or network level battery life ends before receiving patient's condition to the expert. To overcome such situation, we proposed our node level and network level energy efficient system.

\section{BACKGROUND AND RELATED WORK}

Wireless Sensor Networks (WSN) consists of several sensor enable nodes which are distributed in an environment and use batteries as energy resource. These tiny sensor nodes, which consist of sensing, data processing, and communicating components, result in the idea of sensor networks based on collaborative effort of a large number of nodes. Such sensor nodes could be deployed in home, military, science, and industry applications such as transportation, health care, disaster recovery, warfare, security, industrial and building automation, and even space exploration. For system miniaturization, wireless communication, and on-chip signal processing can develop the wireless sensor technology [1], [2]. Nowadays there are rapid increments in deployment of wireless sensor network in real life application [3], [4]. However, energy consumption still remains as a major obstacle for the full deployment and exploitation of this technology, although batteries can be recharged, e.g., solar-energy-harvesting mechanisms [5]. Different approaches introduce by different researchers, such as duty-cycling and data-driven approaches [6], for minimizing energy consumption. Data-driven approaches can be categories into two parts: data compression and energy-efficient data acquisition. As an example, the transmission data is in compressed form by using a variable data length coding method using Walsh function, and it will provide improvement in energy efficiency in signal transmission [8]. In another study, the sensor network was divided into several subsystems, and only high-level inferences are communicated between the subsystems. In this way, the energy consumption for communication decreases as the data to be transmitted decrease [9]. The traffic-adaptive medium access protocol has been designed to reduce energy consumption by allowing sensor nodes to assume a low-power idle state whenever they are not working in transmission or receiving mode [7]. For energy-efficient data acquisition, an adaptive sampling algorithm consisting of duty cycling (the sensor board is switched off between two consecutive samples) and adaptive (the sampling optimal sampling frequency is estimated online) is proposed to reduce energy consumption in a sensor network[10].Researchers have also studied other approaches for energy-aware transmission, including modulation scaling schemes [11], [12], multi hop routing schemes [13], network sectioning [14], [15], and lowpower hardware [16]. Furthermore, a combination of sleep scheduling with block transmission approach has been proposed to achieve energy saving in a wireless multimedia sensor network [17].Motivated by the prior research, an energy-saving strategy consisting of node-level energy saving using adaptive radiofrequency(RF) power setting and network-level energy saving through adaptive network configuration has been proposed in our conference paper[18]. This paper is an extension of[19], in which the periodic sleep/wake-up scheme is added into the sensor node design to further achieve the node-level energy saving. design and implementation of energy efficient sensor node with the new ultralow power microcontroller MSP430F149 being used as the core for the designed sensor node.

The wireless sensor node not only senses the physical capabilities but also it is able to process the obtained data and communicate the results wirelessly. The less energy consumed while data acquisition and processing than communication [13]. Additionally, if each of the sensor nodes consumes energy in network and each component in a sensor node consumes a certain amount of energy, power supply becomes important to ensure proper operation of the entire 
WSN as the number of sensors deployed in a network grows. Hence, constructing effective network structures for the application of WSN with consideration of energy efficiency is of critical importance Energy consumption calculated after sensing the environmental parameters, the results should be transmitted to the central monitoring unit (CMU) or other sensor nodes. For two sensor nodes to communicate, the energy consumption needed for data transmission can be expressed as [20]

$$
\text { ETx }=\text { Ee_tx } \cdot \mathrm{k}+\text { eamp } \cdot \mathrm{d} \alpha,
$$

for a fixed distance, the energy consumed is proportional to the number of data bits. Otherwise, more energy consumed at the longer distance of sensor node and CMU.The sensors are grouped into different clusters, and the obtained data from each sensor node are transmitted to the corresponding cluster head (it is defined as the sensor node that collects the data from others in the cluster). Then, the cluster head will pack the data and transmit them to the CMU.

\section{PROPOSED MODEL}

Projected system involves different sensors MSP430microprocessor.The sensors continuously sense the parameters of patient \& send to MSP430 microprocessor. Processor converts these in to digital form and then these parameters are transmitted to PC by establishing connection (General Packet Radio Service).Some respective technologies to implement wireless network include: Bluetooth, Zigbee, IEE 802.11, CC2500 is highly suitable for implementing wireless network because different sensors are deployed to collect information in well-organized manner.

The designing part includes basically three sections as follows

3.1 Hardware design and softwaredesign

3.2 Systemflowchart

3.3 Design of sensornode
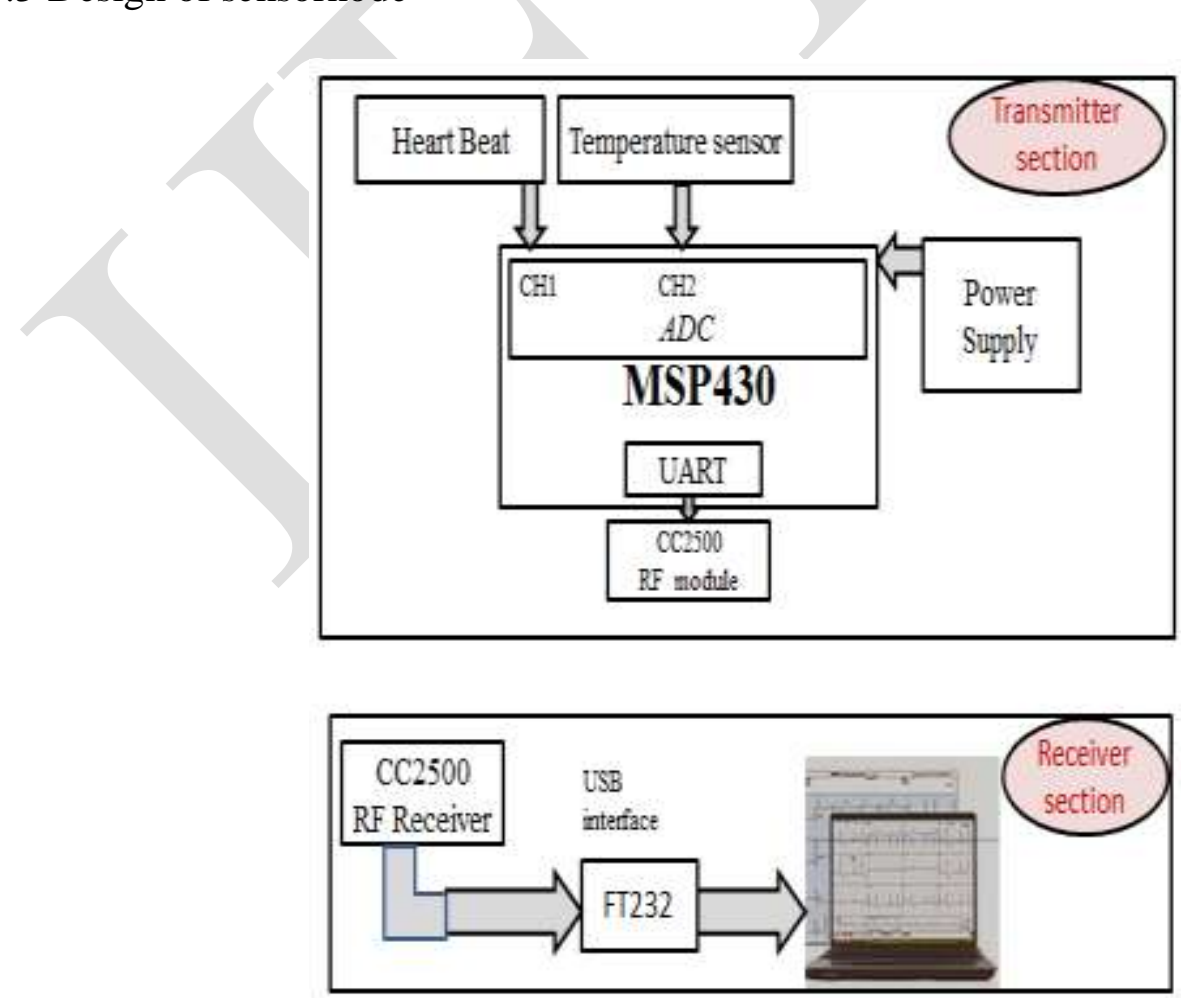

Fig 1:- Block diagram of proposed system 
3.1 Hardware design:

It includes Power supply design, Temperature sensor, Heart beat sensor, UART-232

connector circuit

Power supply circuit:

The hardware of project requires different power supplies.

5 v: for temperature sensor circuit, heartbeat sensor circuit, RS 232 connector circuit.

$1.8 \mathrm{v}$ : for MSP430microprocessor.

$+/-5 \mathrm{v}$ : for heart beat sensor circuit, temperature sensor circuit. Here system is designed by 5 $\mathrm{v}$ power supply using bridge rectifier,

\subsection{System flowchart}

The work flow of this proposed system is as shown in flowchart. Entire scenario is based on mobile sink node and sensor node in the field. In this proposed system the temperature and pulse rate sensor deployed around the patient. Respective sensors sense the physical data and send it to sink node. Mobile sink can travel throughout the field and collect data and stored it on the base station and again the same procedure is repeated but in another round only those sensor sensed data which is previously not available.

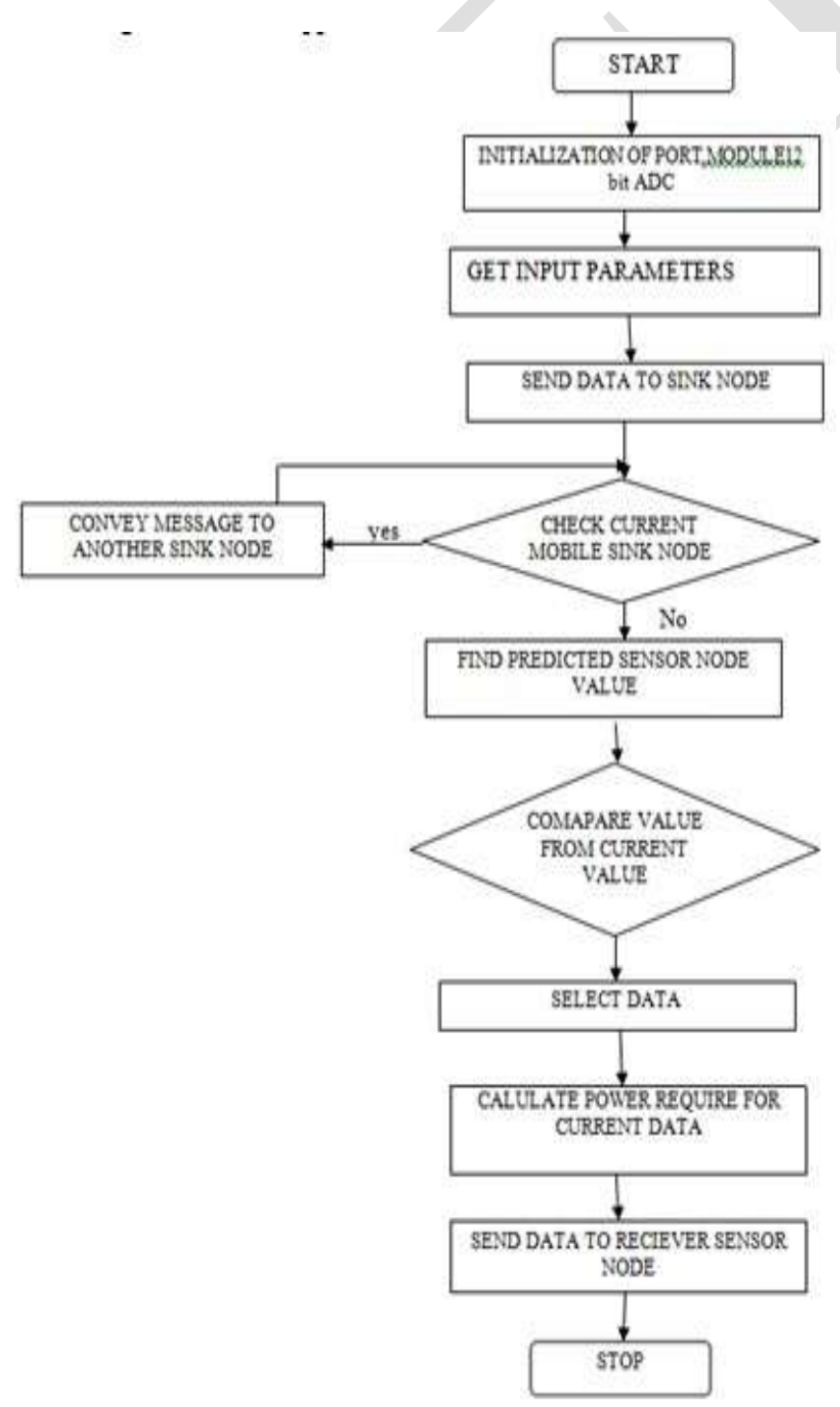

Fig 2 flowchart of system flow 


\subsection{Design of sensor node}

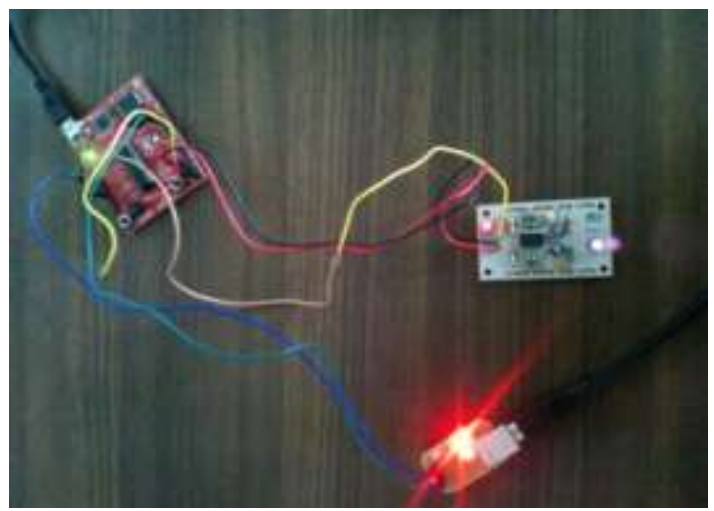

Fig 3:- Design of Sensor Node

In this proposed system, the mobility is provided to the sink node so that the minimum energy required to transmit signal from sensor node to sink node. This can be achieved by using network topology. The mobile sink node can collect data from different physical conditions and send to the central monitoring unit.

\section{RESULT ANALYSIS}

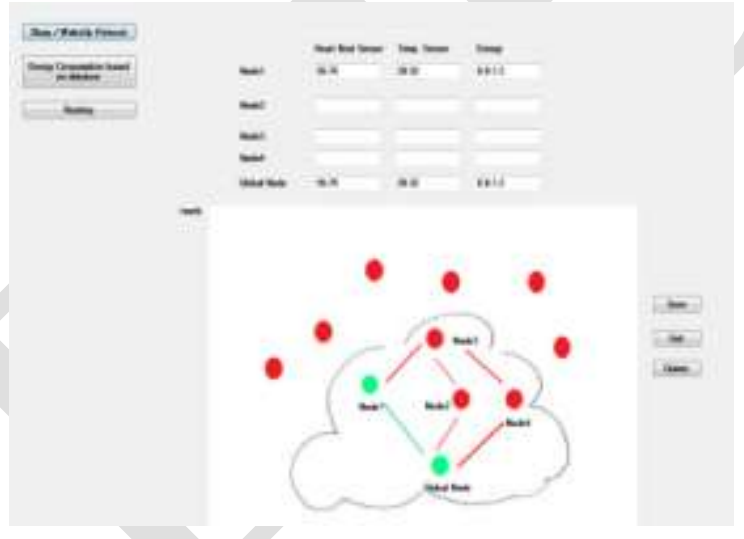

Fig 4 :-Node1 to Global Node Data Transmission

As shown in fig 4 the node1 sends its data to global node by using concept of distance. As distance varies the power is also varies to transmit data. The power is estimated by calculating the power required for temperature sensor, heart beat sensor the Msp430 microcontroller

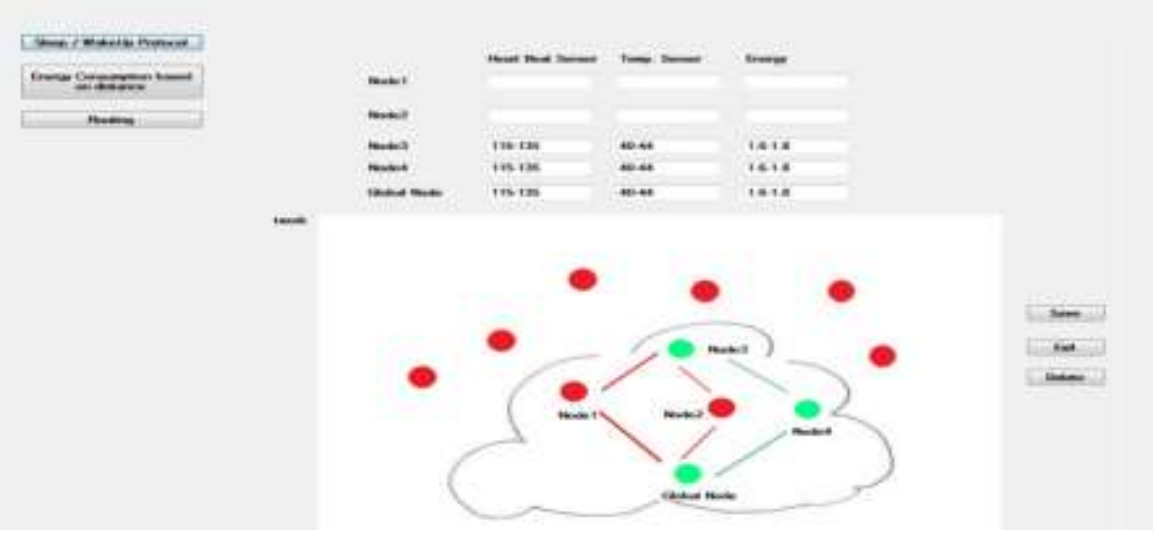

Fig:-5 Node3 to Globle Node Data Transmission through Node4 
As shown in fig 5 the output shows the routing for transmission data. The shortest path chooses by the node 3 for transmitting data to global node. The routing is achieved by the hierarchical routing protocol. In which Node 3 tries to find another node 4 to relay data which may consume less energy than that through directly communication with global node. Since the direction of data transmission can be randomly chosen, various nodes can be chosen, which turn out to cause various energy consumption.

TABLE1 Comparison of Energy consumption and number of node

\begin{tabular}{|l|l|l|l|}
\hline $\begin{array}{l}\text { No. } \\
\text { Nodes }\end{array}$ & Heartbeat & Temperature & Energy \\
\hline Node1 & $55-75$ & $28-32$ & $0.8-1.3$ \\
\hline Node2 & $75-95$ & $32-36$ & $1.2-1.4$ \\
\hline Node3 & $95-115$ & $36-40$ & $1.4-1.6$ \\
\hline Node4 & $115-135$ & $40-44$ & $1.6-1.8$ \\
\hline
\end{tabular}

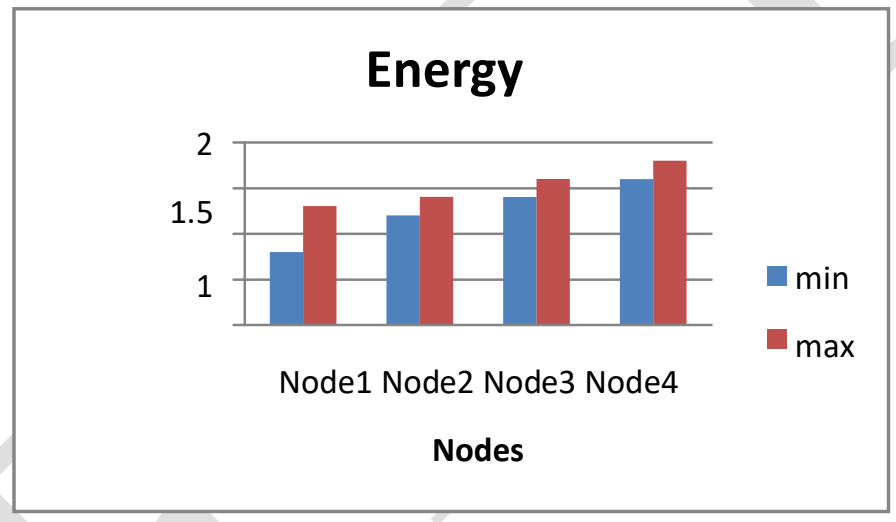

As shown in graph the energy consumption is increases as the distance and number of node increases throughout the network. The energy consumption is calculated in energy consumption of communication/uJ and the varying distance between the nodes and increasing number of nodes while data transmission.

\section{CONCLUSION}

Sink mobility has a very significant impact on the network performance, especially for smart home networks or the networks without fixed infrastructure. In this paper, we have presented the design and implementation of an energy-aware sensor node, which can help in constructing an energy-efficient WSN through mobile sink node which consume maximum energy while signal transmission. The energy saving at mobile sink node is achieved by measuring distance between sensor node and sink node and according to changing distance power is estimated.

\section{References}

1. ZhumingBi, $\|$ An Integrated Environment for Visualization of Distributed Wireless Sensor Networks 2013 10th IEEE International Conference on Control and Automation (ICCA) Hangzhou, China, June 12-14, 2013.

2. Jin Mitsugi, HisakazuHada, Tatsuya Inaba,\|Enabling globally unique sensor ID withdualinterface RF tag\|, 978-1- 4244-9289-3/11/\$26.00 (02011 IEEE.

3. Kaushik Nanda, Kiran Nayak, ShrikrishnaChippalkatti, Ramana Rao, David Selvakumar, 
HaribabuPasupuleti,\| Web based Monitoring and Control of WSN using WINGZ (Wireless IP Network Gateway for Zigbee)\|, 2012 Sixth International Conference on Sensing Technology.

4. Jinsung Byun, Boungju Jeon, Junyoung Noh, Youngil Kim, and SehyunPark, „An Intelligent Self-Adjusting Sensor for Smart Home Services based on ZigBee Communicationsll, IEEE Transactions on Consumer Electronics, Vol. 58, No. 3, August 2012.

5. Chenmin Li, Guoping Tan, Jingyu Wu, Zhen Zhang, LizhongXu, $\|$ Analyzing Cluster-head Selection Mechanisms and Improving the LEACHI, 978-1-4577-0321-8/11/\$26.00 (C)2011 IEEE.

6. G. Chen, J. Branch, M. Pflug, L. Zhu, B. K. Szymanski, -Sense: A Wireless Sensor Network Simulatorll, Advances in Pervasive Computing and Networking, Springer, New York, NY, pp. 249-267,2004.

7. Intelligent Distributed Controls Ltd., -Track, Locate and Monitor Progress in Real Time with ZigBee Networksl, http://www.zigbee.co.uk/, 2009.

8. S. Wielens, M. Galetzka, P. Schneider, -Design Support for Wireless Sensor Networks Based on the IEEE 802.15.4 Standardll, the Proceedings of the IEEE 19th International Symposium on Personal,Indoor and Mobile Radio Communications, pages 1-5, 2008.

9. Stefani, F., Gamba,F.,Goldoni,P.,Savioli,E., Silvestri, A., Toffalini, F,\|REnvDB, a RESTful Database for Pervasive Environmental Wireless Sensor Networksll, IEEE 30th International Conference on Distributed Computing Systems Workshops (ICDCSW), (2010), pp.206 - 2112.

10. Schor,L.,Sommer,P, d Wattenhofer,R.,\|Towards a zeroconfigurationwireless sensor network architecture for smart building\|, Proc. of 1st ACM Workshop on Embedded Sensing Systems for Energy-Efficiency in Buildings, BuildSys f09, Nov. (2009).

11. Guinard,D., Mueller,M., Pasquier-Rocha,J., $\|$ A Resource Oriented Architecture for the Web of Thingsll, 2nd Internet of Things Conference,(2010), Available on IEEE Xplore.

12. Hada,H., Mitsugi,J., IEPC based Internet of Things Architecturel,,IEEE International conference on RFIDTechnology and Applications, September 15-16, (2011).

13. Shelby,Z., Frank,B., and Sturek,D.,|Constrained Application Protocoll, Internet Draft draft-ietf- core-coap, (2010).

14. Sugimoto,K., Mitsugi,J., Nakamura,O., and Murai,J., \|Low power and algile sensor data retrieval using dual interface passive RF tag\|, IEEE RFID-TA, 2010, June.

15. The EPCglobal Architecture Framework Ver.1.4, (2010). [16] WINGZ System Reference Manual Ver.1, CDA C, Bangalore. 